Origins of Life: Self-Organization and/or Biological Evolution? (2009) 79-94

(C) EDP Sciences, 2009

DOI: $10.1051 /$ orvie/2009007

\title{
Elastic growth in thin geometries
}

\author{
J. Dervaux and M. Ben Amar \\ Laboratoire de Physique Statistique de l'École Normale Supérieure, \\ 24 rue Lhomond, 75230 Paris Cedex 05, France \\ e-mail: benamar@Ips.ens.fr
}

\begin{abstract}
Generation of shapes in biological tissues is a complex multiscale phenomenon. Biochemical details of cell proliferation, death and mobility can be incorporated within a continuum mechanical framework by specifying locally the amplitude and direction of growth. For tissues exhibiting an elastic behavior, equilibrium shapes of growing bodies can be evaluated through the minimization of an appropriate energy. This model is applied to thin shells and plates, a geometry relevant to nuts and pollen grains but also leaves, petals and algae.
\end{abstract}

\section{INTRODUCTION}

Morphogenesis describes the set of processes by which a living organism changes its shape. For example, the growth of plants is governed by genetics, atmospheric conditions such as mechanical forces (wind, gravity) or sun exposition, chemical and physical properties of the environment, resulting in a broad and beautiful variety of shapes and functions. All living tissues, both animals and vegetals, share this ability to grow. Evolution of shapes takes place during healthy as well as pathological (e.g. tumors) development. The biological development involves a causal chain of transductions from the genome to the structural level (Green 1996). In particular, the process by which patterns of differentiated cells appear in tissues is well understood. Morphogens are signalling molecules that diffuse within the tissue and produce specific cell response depending on both the concentration (Wolpert 1996; Ashe \& Briscoe 2006) and the slope of the gradient of concentration (Rogulja \& Irvine 2005). They are defined through their function rather than their chemical nature. For example, the morphogen Sonic hedgehog homolog is a protein that controls the development of limbs and spinal cord in mammals (Nüsslein-Volhard \& Wieschaus 1980) whereas retinoic acid is thought to be involved in embryo development of the zebra fish hindbrain (White et al. 2007). In the Drosophila fly, the transcription factor BCD, coded by the gene Bicoïd, is a protein that binds to the DNA of other cells and activates the expression of specific genes (like orthodenticle or hunchback), depending on its concentration (high and low, respectively) (Struhl et al. 1989). Since BCD is secreted locally by a source, gradients of concentration appear and form several regions of differentiated cells, depending on which threshold is reached in every cell by the concentration distribution, introducing an anteroposterior polarity in Drosophila. In this line of thought, and following Turing's seminal work, reaction/diffusion models have been developed to explain the complex emerging patterns of differentiated cells (Cruywagen et al. 1992, 1997; Kondo 2002). In addition to being essential to organogenesis by inducing cellular differentiation, gradients

Article available at http://www.origins-and-evolution.org or http://dx.doi.org/10.1051/orvie/2009007 
of morphogens control cell profileration (Rogulja \& Irvine 2005) and thus ultimately the shape of organs. How the growth of individual cells pushing each others results in the observed macroscopic shape (and the associated residual stress field) is, however, less clear and the object of an active research.

The idea that mechanics play an important role in the shape's determination of vegetal or animal tissues has been raised almost a century ago by the biologist D'Arcy Thompson (1992) and has subsequently raised harsh criticism but also praises (Gould 1971; Dumais 2007). D'Arcy Thompson proposed that evolution alone could not explain macroscopic shapes and that living organisms are subject to physical laws in the same way as inanimate matter: "The waves of the sea, the little ripples on the shore, the sweeping curve of the sandy bay between the headlands, the outline of the hills, the shape of the clouds, all these are so many riddles of form, so many problems of morphology, and all of them the physicist can more or less easily read and adequately solve: solving them by reference to their antecedent phenomena, in the material system of mechanical forces to which they belong, and to which we interpret them as being due .... Nor is it otherwise with the material forms of living things. Cell and tissue, shell and bone, leaf and flower, are so many portions of matter, and it is in obedience to the laws of physics that their particles have been moved, moulded and conformed."

Indeed, the description of the collective behavior of interacting particles is a central question of physics. Due to the highly organized (that is strongly correlated) nature of their numerous components, statistical physics is not much useful, and biological objects are best described within a continuum mechanics framework. When cells and fibers are sparse, that is when they form a suspension (as seen in the circulatory system or the bone marrow) rather than a dense connected network, reaction/diffusion models alone are appropriate because elastic effects can be neglected. On the other hand, for materials that are highly packed with fibres (connective tissues) or which cells are rigid and bound together (epithelial tissues), elasticity must be taken into account. Examination of living tissues from a scale at which they appear continuous (that is above the characteristic length scale of cells, microtubules and other fibers) reveals several macroscopic properties of interest:

- Anisotropy: fibers (elastin, collagen, myofibrils) endow biological tissues with directional properties (Ciarletta et al. 2006, 2008; Ciarletta \& Ben Amar 2009).

- Non-linearity: soft tissues exhibit a typical strain-hardening behavior. An increase of strain comes with an increase of the stiffness. This is also associated with the presence of fibers which elasticity comes into play only when they have uncrumpled, overiding the elasticity of the underlying matrix.

- Incompressibility: a high volume fraction of water results in a high volumetric stiffness. Those properties, reminiscent of those of rubber (Ogden 1997; Treloar 1975; Goriely et al. 2006; Wertheim 1847; Roy 1880), are best described using finite elasticity (Fung 1990, 1993; Skalak et al. 1973; Humphrey 2003). From a macroscopic perspective, growth is a rather slow process (compared to typical mechanical time scales in living tissues) with significant variations taking place in hours or days (Taber 1995). Growth can be localised to surfaces (Skalak \& Hoger 1997) (like accretion at the external surface of a shell) or extends throughout the volume (volumetric growth). While the former case mostly concerns hard tissues (teeth, bones) the latter is characteristic of soft tissues (muscles, tendons, solid tumors, skin). Volumetric growth involves a variation of the 
bulk mass which may or not be compatible with the sample's surroundings (boundary conditions). In addition, adjacent particles of a biological body may evolve at different growth rates (inhomogeneity) and/or in different directions (anisotropy). Thus in order to maintain integrity, that is to prevent the body from overlaping with itself (or with external boundaries) as well as forming holes, stresses are necessary (Ambrosi \& Mollica 2002; Ben Amar \& Goriely 2005; Goriely \& Ben Amar 2005). They are referred to as residual stresses and play a fundamental role in the generation of shapes. Therefore the general idea underlying the physical representation of growth of biological tissues is that the general deformation is due to both growth and elasticity (Hsu 1968; Cowin \& Hegedus 1976; Skalak 1981; Entov 1983; Stein 1995; Drozdov \& Khanina 1997; Hoger 1999).

In all generality, the growth process itself is affected by internal and external loadings. This fact has been known for at least a thousand years as attested by the foot binding custom in medieval China. In the biomechanical literature, this coupling is often referred to as Wolff's law and states that bones develop in response to applied loadings, although this is true for soft tissues as well. It is interesting to note that although the influence of external forces on growth is easily observed and despite a few attempts to derive macroscopic constitutive relationships between local growth rates and stresses (Cowin \& Hegedus 1976; Cowin 1983), the detailed biochemistry underlying this coupling, that is Wolff's law's microscopical counterpart, remains obscure.

To apprehend the growth process macroscopically, it is assumed that the overall deformation can be decomposed in two fundamental steps (Rodriguez et al. 1994): a growth process, assumed as known and possibly incompatible, followed by an elastic relaxation ensuring the compatibility of the body. Those two processes can be represented as tensors, the product of the two being the gradient of the overall deformation. The cumulative growth rates (in the present case, the components of the growth tensor) are the control parameters from which stresses and strains can be calculated using minimization of an appropriate energy. This energy, according to this model, only depends on the elastic part of the forementioned decomposition. Note that this model is not incompatible with the diffusion process. While diffusion models explain what positional information each cell will get and therefore which program of differentiation and proliferation the cell will start, the elastic model aims at describing how this individual program translates at a larger scale. In this respect, several authors have attempted to couple the diffusion process with the elasticity of the tissue in a mixture theory (Humphrey \& Rajagopal 2002).

This paper is devoted to illustrate this macroscopic theory of elastic growth in two simple geometries: a shell and a plate. We shall see that, whatever the biochemical details governing the growth process are, the resultant shape and stresses of a simple growing biological body can be derived from minimization of an elastic energy.

In a first part we briefly introduce the necessary tools of finite elasticity that we shall use later on, as well as the multiplicative decomposition of the gradient deformation tensor.

In a second part, we study the growth of shells under external pressure. This is relevant to spherical connective solid tumors for example. In those cases, growth takes place at the outer shell of the tumor, leaving a core of necrosed cells inside. In the botanic realm, several examples of shells are also observed : pollen grains possess an external hard spherical shell (exine) enclosing a few soft cells while nuts also present a hard nutshell enclosing the fruit. This geometry allows us to investigate how the material non-linearity 
and geometry can influence the stability of growing shells. The large homogeneous anisotropic growth rates lead to an increase in the thickness on one hand and emergence of residual stresses that pile up on the other hand. The competition between those geometrical and mechanical effects can either stabilize or destabilize the shell depending on its thickness. Moreover, this stability depends on the constitutive equation describing the material. A rather counterintuitive result is the fact that strain-hardening effect can decrease the stability of shells.

In a third part, we focus on thin hyperelastic plates. This geometry is widely encountered in nature, since petals or leaves are flat tissues and have been the subject of several studies (Coen et al. 2004; Rolland-lagan et al. 2003; Green 1996; Newell \& Shipman 2005). Furthermore, a variety of cancers originate from the stem cells of the epithelial tissue, usually a thin layer of cells linked to a thick soft connective tissue, so this geometry is relevant to tumor growth. In the limit of small thickness (compared to the lateral dimensions of the plate) and moderate deflections (in a sense to be precised later), the equilibrium equations are dominated by geometrical effects and are independent of the constitutive equation. Growth acts as a source of curvature and can induce non-trivial shapes. When those shapes are not available physically (they may exist mathematically), buckling occurs to release the residual stresses, leading to morphogenesis (Dervaux \& Ben Amar 2008; Dervaux et al. 2009; Müller et al. 2008).

\section{ELEMENTS OF FORMALISM AND NOTATIONS}

Referring to the classical formulation of nonlinear elasticity, we consider an elastic body $\mathcal{B} \in \Re^{3}$ in the reference configuration $\Omega_{0}$. The deformation can be defined as an objective mapping $\chi: \Omega_{0} \rightarrow \Omega$ that transforms the material point $\mathbf{X} \in \Omega_{0}$ to a position $\mathbf{x}=\boldsymbol{\chi}(\mathbf{X}, \mathrm{t})$ in the current configuration $\Omega$. Defining the geometric deformation tensor by $\mathbf{F}=\partial \mathbf{x} / \partial \mathbf{X}$, a multiplicative, also called Kröner-Lee, decomposition has been proposed to incorporate the growth process (Rodriguez et al. 1994):

$$
\mathbf{F}=\mathbf{A G}
$$

where $\mathbf{G}$ is referred to as the growth tensor, and $\mathbf{A}$ as the elastic deformation tensor, that represents the purely elastic contribution needed to maintain the overall compatibility of the mapping. Since the material is elastically incompressible, then we have $\operatorname{det} \mathbf{A}=1$ and $J=\operatorname{det} \mathbf{F}=\operatorname{det} \mathbf{G}$ describes the local change in volume due to growth. The model states: (i) that there exists a zero-stress reference state, $(i i)$ that the geometric deformation gradient $\mathbf{F}$ admits a multiplicative decomposition in the form of Eq. (2.1), and (iii) that the response function of the material only depends on the elastic part of the total deformation. Some subtle aspects of this formulation have been discussed (Lubarda 2004; Goriely \& Ben Amar 2007) but we shall point out that this theory of finite elastic growth treats the main features of the growth process: large changes of volume, anisotropy of growth, emergence of residual stresses and even time-dependent processes. Since growth is mathematically represented by a tensor, this formalism incorporates easily the spatial inhomogeneities as well as the anisotropy of growth, which is essential for plants (Coen et al. 2004). Moreover, as we consider very slow growth phenomena compared to elastic or viscoelastic relaxation, the system will be at the elastic equilibrium even if the tensors are time-dependent. Consider a free homogeneous elastic body with zero stress in its 
reference configuration and let it undergo a homogeneous isotropic growth (that is spaceindependent). In that case, there exists an affine mapping describing the shape change, i.e. it is a well defined deformation which does not introduce any overlap or cavitation and there is no need for an elastic accommodation of the body. This situation is atypical and only arises when the growth tensor defines a deformation and therefore a current, stress-free, configuration. In the general case, the growth tensor is not the gradient of a deformation and an elastic process is needed (which is not a deformation either), consequently inducing residual stresses within the material even in the absence of external loading. In that case the grown "state" cannot be physically achieved (it is why we do not use the word configuration). However, the third point states that if the stress happens to be zero at a material point of the body, then the value of the growth tensor at that point is equal to the geometric deformation tensor. This provides the conceptual tool to build the grown "state": by applying external loads to the body in the current configuration, one can locally reduce the stress to zero at a material point (but not in the whole body), the growth tensor is then equal to the value of the geometric deformation tensor at that point. That is there is locally a deformation that describes the grown "state", which is therefore a collection of configurations. For that reason, the growth tensor $\mathbf{G}$ and the elastic tensor A are sometimes referred to as local deformation tensors.

These assumptions allow to define an hyperelastic strain energy function $\mathcal{W}$ for the material body, as a function of the tensor $\mathbf{A}=\mathbf{F} \mathbf{G}^{-1}$. In the following we focus on incompressible homogeneous isotropic materials, so that the strain energy function will only depend on the two first invariants $\mathcal{I}_{1}$ and $\mathcal{I}_{2}$ of the right Cauchy-Green elastic deformation tensor $\mathbf{C}=\mathbf{A}^{t} \mathbf{A}$. Furthermore, under the regularity assumption that $\mathcal{W}$ is continuously differentiable infinitely many times with respect to $\mathcal{I}_{1}, \mathcal{I}_{2}$, we can write $\mathcal{W}$ as:

$$
\mathcal{W}\left(\mathcal{I}_{1}, \mathcal{I}_{2}\right)=\sum_{k, l=0}^{\infty} c_{k l}\left(\mathcal{I}_{1}-3\right)^{k}\left(\mathcal{I}_{2}-3\right)^{l}
$$

So that the strain-energy function is entirely determined by the values of the coefficients $c_{k l}$. Note that several strain-energy functions commonly used in the literature only involve the first coefficients of this development, $c_{01}$ and $c_{10}$. This is the case of the Mooney-Rivlin and Neo-Hookean models for example. Since we consider elastically incompressible materials we have to consider the scalar relationship $C(\mathbf{A})=\operatorname{det} \mathbf{A}-1=0$, so that the nominal stress tensor $\mathbf{S}$ (also called first Piola-Kirchhoff tensor) can be written as:

$$
\mathbf{S}=J \frac{\partial \mathcal{W}}{\partial \mathbf{F}}-p J \frac{\partial C(\mathbf{A})}{\partial \mathbf{F}}=J \mathbf{G}^{-1} \frac{\partial \mathcal{W}}{\partial \mathbf{A}}-p J \mathbf{G}^{-1} \mathbf{A}^{-1}
$$

The second Piola-Kirchhoff tensor $\boldsymbol{\sigma}$, which is the force mapped to the undeformed configuration on undeformed area, is defined as $\sigma=\left(\mathbf{F}^{-1}\right)^{t} \mathbf{S}$. Note that the nominal stress tensor is not symmetric, while the second Piola-Kirchhoff tensor is symmetric, being $\boldsymbol{\sigma}=J \partial \mathcal{W} / \partial(\mathbf{C})-p J \mathbf{C}^{-1}$. The Cauchy stress tensor $\mathbf{T}$, which gives the stress after deformation in the current configuration, is found through the geometric connection:

$$
\mathbf{T}^{\mathrm{t}}=J^{-1} \mathbf{F S}=\mathbf{A}\left(\frac{\partial \mathcal{W}}{\partial \mathbf{A}}-p \frac{\partial C(\mathbf{A})}{\partial \mathbf{A}}\right)
$$


In order to express the stress tensors in terms of the invariants $\mathcal{I}_{1}$ and $\mathcal{I}_{2}$, let us consider the following relations:

$$
\frac{\partial \mathcal{I}_{1}}{\partial \mathbf{A}}=2 \mathbf{A}^{\mathrm{t}} \quad \text { and } \quad \frac{\partial \mathcal{I}_{2}}{\partial \mathbf{A}}=2\left(\mathcal{I}_{1} \mathbf{I}-\mathbf{A}^{\mathrm{t}} \mathbf{A}\right) \mathbf{A}^{\mathrm{t}} .
$$

The Cauchy stress, from Eqs. $(2.4,2.5)$, can be rewritten as:

$$
\mathbf{T}=2 \frac{\partial \mathcal{W}}{\partial \mathcal{I}_{1}} \mathbf{A} \mathbf{A}^{\mathrm{t}}+2 \frac{\partial \mathcal{W}}{\partial \mathcal{I}_{2}}\left(\mathcal{I}_{1} \mathbf{A} \mathbf{A}^{\mathrm{t}}-\mathbf{A} \mathbf{A}^{\mathrm{t}} \mathbf{A} \mathbf{A}^{\mathrm{t}}\right)-p \mathbf{I} .
$$

so that $\mathbf{T}^{\mathrm{t}}=\mathbf{T}$, which is the local form of Cauchy's second law of motion for the balance of rotational momentum. We also define the Green tensor $\mathbf{E}$ as $\mathbf{E}=(1 / 2)\left(\mathbf{A}^{\mathrm{t}} \mathbf{A}-\mathbf{I}\right)$.

\section{SHELLS}

Consider a growing hyperelastic incompressible shell under applied loads. We work with spherical coordinates denoted by $(R, \Theta, \Phi)$ in the reference configuration. Let us denote the inner and outer radii of the spherical shell by $R=A$ and $R=B$ in the reference configuration. After deformation, the shell is now descibed by its radius $r=r(R)$. Assume the growth process is axially symmetric, the tensor $\mathbf{G}$ can be written:

$$
\mathbf{G}=\operatorname{diag}\left(\gamma_{1}, \gamma_{2}, \gamma_{2}\right) \text {. }
$$

Isotropic growth is achieved when $\gamma_{1}=\gamma_{2}$. When $\gamma_{2}>\gamma_{1}$, growth is circumferential whereas $\gamma_{1}>\gamma_{2}$ is referred to as radial growth. The relevant growth parameter is therefore $\gamma=\gamma_{1} / \gamma_{2}$. Accordingly, the geometric deformation tensor is given by:

$$
\mathbf{F}=\operatorname{diag}\left(r^{\prime}, r / R, r / R\right) .
$$

According to the multiplicative decomposition, the elastic strain tensor reads:

$$
\mathbf{A}=\operatorname{diag}\left(\alpha_{1}, \alpha_{2}, \alpha_{2}\right) \text {. }
$$

Incompressibility condition yields the following consequences: $\alpha_{2}=\alpha, \alpha_{1}=\alpha^{-2}$ and, using $\mathbf{F}=\mathbf{A}$.G, we get that $r^{\prime}=\alpha^{-} 2 \gamma_{1}$ and $r / R=\alpha \gamma_{2}$. Combining the last two relations and integrating in the radial direction gives:

$$
r^{3}-a^{3}=3 \int_{A}^{R} \gamma_{1} \gamma_{2}^{2} R^{2} \mathrm{~d} R .
$$

Assuming the growth process is homogeneous, this relation can be integrated:

$$
\alpha^{3}=\gamma\left(1-\frac{A^{3}}{R^{3}}\right)+\frac{a^{3}}{\gamma_{2}^{3} R^{3}}
$$

Without loss of generality, we can take the internal radial stress $T_{R}(A)=0$ and $T_{R}(B)=$ $-P$. If $P>0$ the shell is subject to an external pressure otherwise the shell is inflated with an internal pressure $-P$. This boundary condition yields:

$$
-P=\gamma \int_{\alpha_{a}}^{\alpha_{b}} \frac{\partial_{\alpha} \mathcal{W}}{\left(\gamma-\alpha^{3}\right)} \mathrm{d} \alpha
$$

where $\mathcal{W}$ is the strain energy function. The last two relations allow one to find the inner strain $\alpha_{a}$ and thus the overall deformation $r(R)$. 

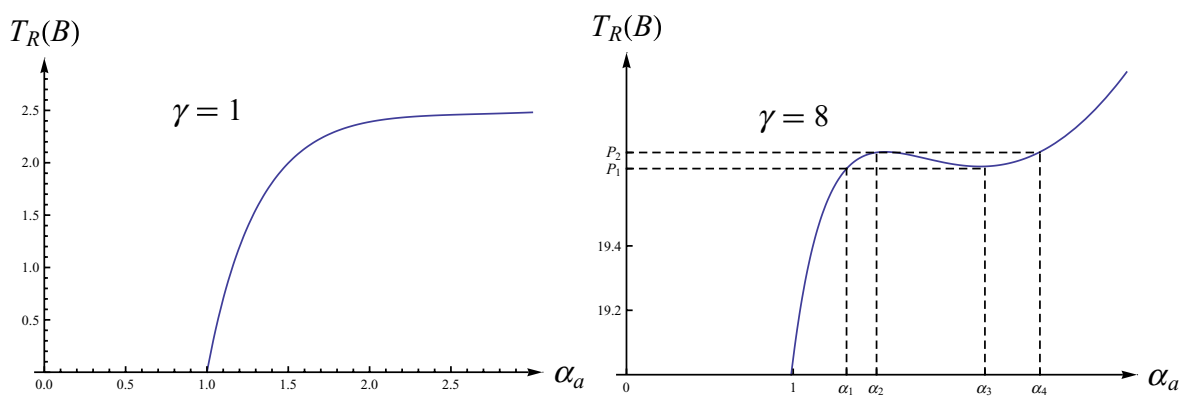

Figure 1. When the growth parameter is high enough (meaning the growth process is largely anisotropic), an inflation jump can occur. Here are shown the stress-strain curves for different values of $\gamma$. Left: for $\gamma=1$, the curve is strictly concave and the inner strain $\alpha_{a}$ increases monotically with increased pressure. Right: for $\gamma=8$, an increase in pressure yields a discontinuous increase of $\alpha_{a}$.

\subsection{Inflation jump}

An interesting effect that can be induced by growth is an inflation jump. This hysteresis effect arises when the stress $T_{R}(B)$ as a function of the inner stretch $\alpha_{a}$ loses its monoticity and present both a local minimum and a local maximum at finite stretch. In the case of a Mooney-Rivlin material, the strain-energy function can be written $\mathcal{W}=$ $\mu_{1}\left(\alpha_{1}^{2}+\alpha_{2}^{2}+\alpha_{3}^{2}-3\right)+\mu_{2}\left(\alpha_{1}^{-2}+\alpha_{2}^{-2}+\alpha_{3}^{-2}-3\right)$. As represented in figure 1 , when the pressure is increased and reached the value $P_{2}$, the inner strain " "jumps", from $\alpha_{2}$ to $\alpha_{4}$. Conversely, when the pressure is reduced to $P_{1}$, the strain goes from $\alpha_{3}$ to $\alpha_{1}$.

\subsection{Bifurcation}

We now consider the stability of a shell with respect to infinitesimal deformations. The general procedure for solving the equilibrium equations around the grown state obtained previously involves the expansion of the solution in spherical harmonics and resolution for each mode. Two simplifications arise: firstly, the displacement in the direction $\mathbf{u}_{\phi}$ decouples from the two other directions and does not play a role in the determination of the bifurcation value. In addition, the equations of mechanical equilibrium are identical for each mode of the spherical harmonics so the analysis can be restricted to the order 0 (no dependance on $\phi$ ). Consequently, we focus on axisymmetric perturbation that can be written:

$$
\chi^{(1)}=[u(r, \theta), v(r, \theta), 0]
$$

Upon separation of variables into a function of $r$ alone and a Legendre polynomial in $\cos (\theta)$, the linear stability analysis yields a fourth order linear differential equation in $r$. In the Neo-Hookean case, its coeficients are function of $r$ and of the growth parameter $\gamma$. We do not present the details of the calculation but rather summarize the main results. In the absence of growth, note that internally pressurized shells $(P<0)$ made of neoHookean material are always stable whereas external pressure $(P>0)$ may destabilize the shell above a critical value. As a consequence, emphasis has been put on externally 

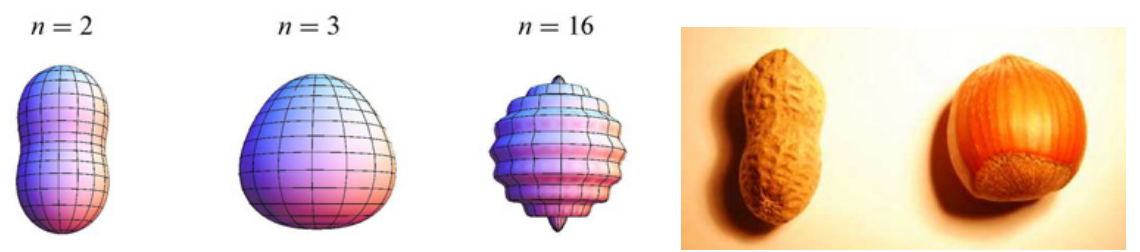

Figure 2. Left: some destabilized modes. Note that the amplitude and the sign of the perturbation is arbitrary and not given by the perturbation analysis. Right: peanut and hazelnut (photography by Elsa Bayart).

pressurized shells. When constant growth is taken into account, destabilized modes are not ordered, and the selection of the first destabilized mode depends on both the geometry and $\gamma$. Figure 2 illustrates the aspect of a few selected modes.

In particular, there is a competition between the increase in thickness induced by growth which stabilize the shell and the buid-up of compressive residual stress that tends to destabilize it. For thick shells, the increase in thickness (radial growth: $\gamma>1$ ) does not affect by a large extent the value of the critical strain needed for the instability to appear. Piling up of residual stresses is the leading effect and thick shells are more prone to become unstable. On the other hand thin shells are strenghtened by an increase of the thickness which drastically increases the threshold of the instability, making them harder to destabilize.

The result that geometric effects prevail over the behavior of thin shells brings up the possibility of some simplifications of the equilibrium equations for thin objects. Indeed, the critical pressure for axisymetric instability depends weakly on the specific strainenergy function used for the calculation in the limit of thin shells (see figure 3). This means that, in this limit, material nonlinearity is a mechanical effect of a lesser magnitude than geometrical effect for large deformations. This suggests that a theory of plates (for simplicity, although a similar theory of shells can be considered) can be formulated in which the material nonlinearity appears as a second-order effect if the deformations are "not too large". Starting from three dimensional nonlinear elasticity, several models of thin plates can be derived, depending on the scaling of the strain field. Using the Föppl-von Kármán (FvK) scalings, we found that the specific constitutive relationship (generalized Hooke's law), which is assumed in the usual derivation of the FvK model, is in fact valid for all materials and follows from the small thickness hypothesis, thus greatly extending the range of validity of the FvK model. The FvK model was derived independently by Föppl (1907) and von Kármán (1907) and is now a classical topic in Hookean elasticity (Landau 1990; Ciarlet 1980). The behavior of such a plate thus involves the geometry, the instantaneous elastic response of the plate (the elastic coefficient for infinitesimal strain) and, in our model, the growth process. Neglecting the material nonlinearity allows an in-depth analysis of the effect of growth.

In the next section, we outline the derivation of the model and, as an illustration, we present an example of a growing biological system: the thin cap of a green algae: acetabularia acetabulum. 

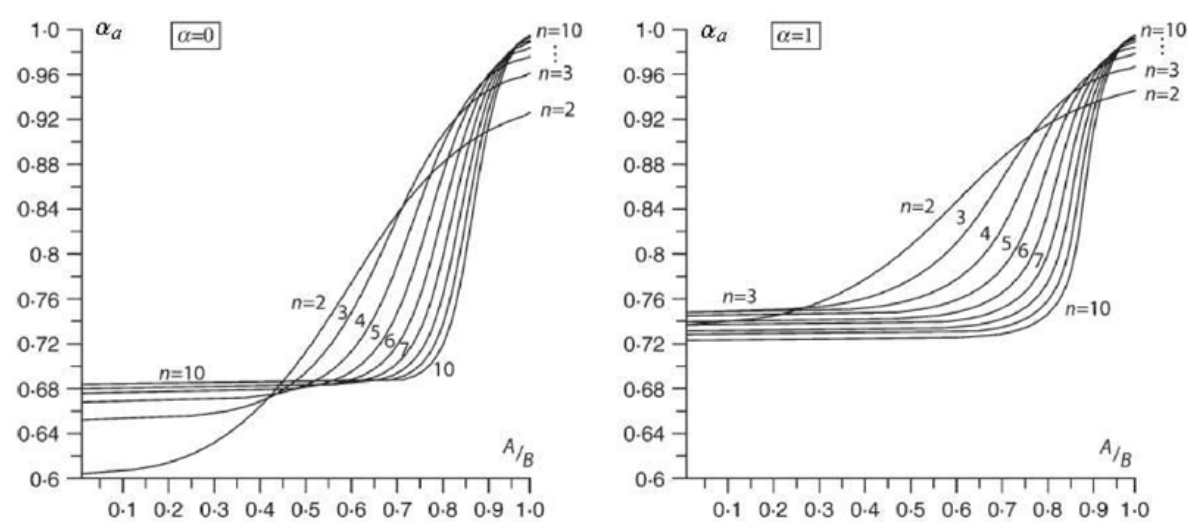

Figure 3. For each mode, the critical value of the stretch $\alpha_{a}$ at which the instability occurs is plotted for a Fung material. The strain-energy function for such a material is given by: $\mathcal{W}=$ $\frac{1}{2 \alpha}\left(\exp ^{\alpha\left(\alpha_{1}^{2}+\alpha_{2}^{2}+\alpha_{3}^{2}-3\right)}-1\right)$. In the limit $\alpha \rightarrow 0$, the material is Neo-Hookean. The parameter $\alpha$ is a measure of the strain-hardening effect. For thick shells $(A / B \ll 1)$, a change in the thickness does not affect the critical strain by a large extent whereas thin shells are greatly stabilized by an increase in the thickness. In addition it can be seen on those curves that the critical strains for each mode weakly depend on the parameter $\alpha$ in the limit of thin shells, by contrast with thick shells.

\section{PLATES}

\subsection{The model}

We consider an initially rectangular plate of width $\left(L_{X}, L_{Y}\right)$, both of same order $L$ and of thickness $H \ll L$. We use Cartesian coordinates in which the place of each material point is given by $\mathbf{X}=X \mathbf{e}_{X}+Y \mathbf{e}_{Y}+Z \mathbf{e}_{Z}$ with the convention that $(0,0,0)$ is the center of the middle surface of the sample. The deformation of the sample is described by a displacement field:

$$
\mathbf{u}=U(X, Y, Z) \mathbf{e}_{X}+V(X, Y, Z) \mathbf{e}_{Y}+(W(X, Y, Z)+\zeta(X, Y)) \mathbf{e}_{Z},
$$

$\zeta(X, Y)$ being the displacement of the middle surface compared to which $W$ is small. We assume that the components of the displacement fields are small compared to the characteristic lateral size of the sample $L$ but may be large compared to the thickness $H$. The boundary condition at the border of an elastic body states that the Cauchy stress $\mathbf{T}$ is equal to the applied external pressure $P$. The in-plane stresses inside the plate being typically much larger than the applied pressure and the curvature of the surface being small, we consider that $\mathbf{T} . \mathbf{e}_{Z}=0$. In addition this relation is assumed to be valid throughout the sample. This assumption is referred to as the membrane assumption and is also applied in the description of stretched tissues for which the bending is neglected (membrane theories) (Haughton 2001). Assuming the cumulative growth rates are independent of $Z$, to the leading order in the sheet's thickness, we get for the in plane displacement:

$$
U=-Z\left(\frac{\partial \zeta}{\partial X}-g_{13}-g_{31}\right)+U_{0}(X, Y),
$$




$$
V=-Z\left(\frac{\partial \zeta}{\partial Y}-g_{23}-g_{32}\right)+V_{0}(X, Y)
$$

the third relation giving the internal pressure $p$. The FvK scalings require $U_{0}$ and $V_{0}$, the in-plane displacements of the middle surface, to be of order $\zeta^{2} / L$. For consistency, the components $g_{3 \alpha}$ and $g_{\alpha 3}$ are of order $\zeta / L$ whereas the other components scale like $\zeta^{2} / L^{2}$. Moreover, the increase in thickness $g_{33}$ is neglected in our model since, being independent of $Z$, it does not introduce any incompatible displacement. The incompressibility assumption allows to eliminate the function $W(X, Y, Z)$ by expressing it in terms of $\zeta, U_{0}$ and $V_{0}$, which are function of $X$ and $Y$ only. The problem is therefore reduced to the study of a two dimensional surface. On this surface, the stress field is related to the strain through the constitutive relationship:

$$
\sigma_{\alpha \beta}=4\left(\frac{\partial \mathcal{W}}{\partial \mathcal{I}_{1}}+\frac{\partial \mathcal{W}}{\partial \mathcal{I}_{2}}\right)\left(E_{\alpha \beta}-E_{Z Z} \delta_{\alpha \beta}\right)=\frac{2 E_{Y o u n g}}{3}\left(E_{\alpha \beta}-E_{S S} \delta_{\alpha \beta}\right)
$$

where $\delta_{\alpha \beta}$ is the Kronecker delta symbol. This relationship is a generalized Hooke's law. Furthermore, the Green tensor can be written:

$$
E_{\alpha \beta}=\frac{1}{2}\left(\frac{\partial U_{\alpha}}{\partial X_{\beta}}+\frac{\partial U_{\beta}}{\partial X_{\alpha}}+\frac{\partial \zeta}{\partial X_{\alpha}} \frac{\partial \zeta}{\partial X_{\beta}}-g_{\alpha \beta}-g_{\beta \alpha}-g_{3 \alpha} g_{3 \beta}\right) .
$$

Let us outline the meaning of those two expressions. The relation (4.4) is valid whatever the strain-energy function. Under the range of displacement considered, all materials behave in the same way (up to an elastic coefficient) (Erbay 1997). Therefore the determination of the particular strain-energy function describing a material, which is not a simple experimental procedure for biological samples, is not necessary. Furthermore, it follows from (4.5) that the multiplicative decomposition (2.1) reduces to an additive one for the Green tensor. Such an additive decomposition has been widely used in growth problem of thin samples (Audoly 1999; Audoly \& Boudaoud 2003), albeit with no theoretical justification. In particular, this makes the concept of target metric (Sharon et al. 2002; Marder \& Papanicolaou 2006; Marder et al. 2007; Klein et al. 2007; Efrati et al. 2008) compatible with the present model.

In a second step, the energy density is expanded in powers of $Z$. After integration in the $Z$ direction, the first two terms of this expansion are kept, yielding a term in $H$, called the stretching contribution and a contribution in $H^{3}$ representing the bending part of the energy. The first term of this surface energy density is of order $E_{\text {Young }} H \zeta^{4} / L^{4}$ while the bending contribution is of order $E_{\text {Young }} H^{3} \zeta^{2} / L^{4}$. The two contributions are of the same order when the out-of-plane displacement $\zeta$ is of order $H$. Equilibrium equations are obtained by equating to zero the variational derivative of the energy with respect to the three components of the displacement field and read:

$$
\begin{aligned}
D\left(\Delta^{2} \zeta-\Delta C_{M}\right)-H \frac{\partial}{\partial X_{\beta}}\left(\sigma_{\alpha \beta} \frac{\partial \zeta}{\partial X_{\alpha}}\right) & =P \\
\frac{\partial \sigma_{\alpha \beta}}{\partial X_{\beta}} & =0
\end{aligned}
$$


where $C_{M}=\frac{\partial}{\partial X}\left(g_{31}+g_{13}\right)+\frac{\partial}{\partial Y}\left(g_{32}+g_{23}\right)$ is an induced mean curvature and $P$ is the external pressure $D=\frac{E_{Y o u n g} H^{3}}{9}$. The first equation of the previous system is the balance of rotational momentum while the next two are the equilibrium of in-plane forces. This system of equations can also be reformulated using the Airy potential function $\chi$ which is the elastic analog of the current function in hydrodynamics. The Airy potential is defined by:

$$
\sigma_{X X}=\frac{\partial^{2} \chi}{\partial Y^{2}}, \quad \sigma_{X Y}=-\frac{\partial^{2} \chi}{\partial Y \partial X}, \quad \sigma_{Y Y}=\frac{\partial^{2} \chi}{\partial X^{2}} .
$$

This function fulfills the in-plane equilibrium equations and thus a specific equation for $\chi$ must be derived. This is achieved by using the constitutive relationship (4.4). In this case, the system of equations can be written:

$$
\begin{aligned}
& D\left(\Delta^{2} \zeta-\Delta C_{M}\right)-2 H[\chi, \zeta]=P \\
& \Delta^{2} \chi+E_{\text {Young }}\left([\zeta, \zeta]-C_{G}\right)=0
\end{aligned}
$$

where the bracket $[.,$.$] is defined through:$

$$
[a, b]=\frac{1}{2} \frac{\partial^{2} a}{\partial X^{2}} \frac{\partial^{2} b}{\partial Y^{2}}+\frac{1}{2} \frac{\partial^{2} a}{\partial Y^{2}} \frac{\partial^{2} b}{\partial X^{2}}-\frac{\partial^{2} a}{\partial X \partial Y} \frac{\partial^{2} b}{\partial X \partial Y},
$$

and the function $C_{G}$ is given by the following expression:

$$
C_{G}=-\frac{\partial^{2}}{\partial Y^{2}}\left(g_{11}+g_{31}^{2}\right)-\frac{\partial^{2}}{\partial X^{2}}\left(g_{22}+g_{32}^{2}\right)+\frac{\partial^{2}}{\partial X \partial Y}\left(g_{12}+g_{21}+g_{31} g_{32}\right) .
$$

The function $C_{G}$ corresponds to the Gaussian curvature of a surface whose metric is given by $\mathrm{d} x^{2}=G_{\alpha \beta} G_{\alpha \gamma} \mathrm{d} X_{\beta} \mathrm{d} X_{\gamma}$ (to leading order). Those equations are a generalization of the FvK system to which they reduce in the absence of growth $\left(C_{G}=C_{M}=0\right)$. Since $C_{G}$ is a Gaussian curvature and $C_{M}$ the $Z$ component of the divergence of a tensor, they are independent of the choice of coordinates. In general, growth induces a mean and a Gaussian curvatures. When there exists a physical surface with such curvatures (compatible with the boudary conditions), it is a stress-free $(\chi=0)$ minimum of the elastic energy. However this is highly unlikely and most of the time residual stresses appear $(\chi \neq 0)$. The physical surface is therefore "close", to the prescribed surface and buckling may occur to realease the residual stress. Also of interest is the limiting case of large deformations $(\zeta \gg H)$, for which it is possible to discard the bending contribution. In this case, the equilibrium surface is a surface of prescribed Gaussian curvature, that is, a Monge-Ampère problem $\left([\zeta, \zeta]=C_{G}\right)$ for which a vast literature exists. In the abscence of growth $\left(C_{G}=0\right)$, the problem reduces to the study of surfaces of zero Gaussian curvature (developable surfaces) and has led to the elegant folds theory (Ben Amar \& Pomeau 1997; Cerda \& Mahadevan 1998; Guven \& Müller 2008). Once a family of solution to the Monge-Ampère solution is found, the physical solution is selected through minimization of the bending energy.

\subsection{Buckling instability}

In order to investigate the effect of growth anisotropy, the circular geometry is especially well suited. Indeed, in polar coordinates $(R, \Theta)$, an anisotropic diagonal growth tensor represents an incompatible growth process. For this purpose, consider a disk of initial 

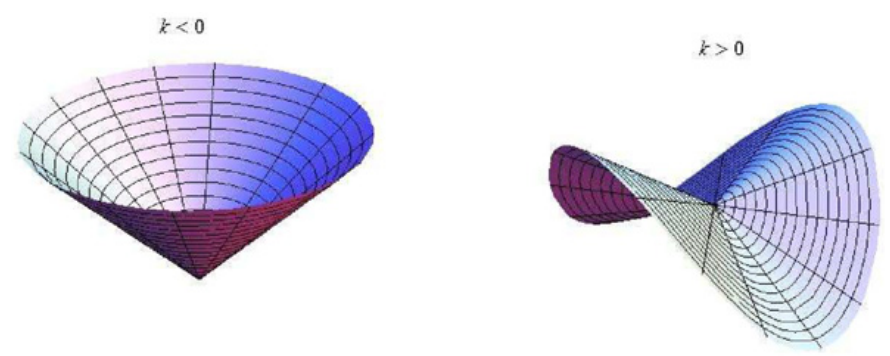

Figure 4. Plot of the solution of the Föppl-von Kármán equations in the limit of large deformations. It is interesting to note that far above the threshold, the selected modes are characterized by the same wavelength as the modes obtained at the threshold of instability.

radius $R_{i}$ and a growth tensor of the form $\mathbf{G}=\operatorname{diag}\left(1+g_{1}, 1+g_{2}, 1\right)$. Within the range of deformations we consider, the relevant growth parameter is $k=g_{2}-g_{1}$. As previously mentionned, the growth in the direction transverse to the disk is irrelevant. If $k \neq 0$, residual stresses appear. Assuming stress-free boundary conditions, there exists a solution of the equilibrium equations in which the only non-zero component of the stress tensor $\sigma$ is $\sigma_{\theta \theta}=-2 k E_{Y o u n g} / 3$. Thus the stress is uniform and the only non-zero component of the deformation field is $U_{R}(R)=(2 R / 3)\left(g_{2} / 2+g_{1}\right)$. When circumferential growth is prevailing, the residual stress is compressive, whereas a tensile stress arise when growth is mainly radial. Linear stability analysis yields the critical anisotropy ratio necessary for buckling to occur. Of course the value of this critical ratio differs depending on the sign of $k$. For circumferential growth (or equivalently radial resorption), the critical value is found to be $k_{c}=3.08 H^{2} /\left(6 R^{2}\right)$, leading to a saddle shape. Alternatively, radial growth induces a conical shape above a threshold of $k_{c}=-7.82 H^{2} /\left(6 R^{2}\right)$. At large deformations, solutions can also be found using the procedure outlined in the previous paragraph. Neglecting the bending term, the general solution of the Monge-Ampère equation is a cone. Minimization of its bending energy leads to a cone of revolution for negative $k$ and a cone with two oscillations for positive $k$. Those solutions are represented in the figure 4.

This simple model turns out to describe quite accurately the morphological changes undergone by the cap of the acetabularia acetabulum algae. Experiments on a population of this very simple unicellular organism show that radial growth dominates in the early stage of their development while circumferential growth prevails later (Serikawa \& Mandoli 1998). Indeed, their caps evolve from a conical to a flat (a short transition of isotropic growth) to a saddle shape.

At much larger deformations, this plates theory is not valid anymore. However, minimization of the bending energy of conical surfaces leads in a first time to larger folds, and secondly to the apparition of a larger number of folds in order to prevent self contact, energetically costly (Müller et al. 2008). The resulting shapes are strongly reminiscent of the oscillatory shape of black mushrooms as seen in figure 5 .

\section{CONCLUSION}

Even though simplistic from a biological standpoint, the elastic theory of growth allows the detailed investigation of the morphological changes induced by a given simple 


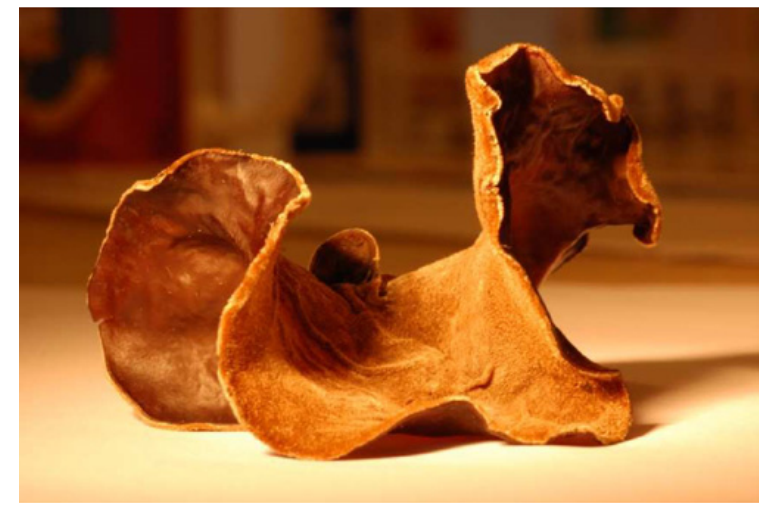

Figure 5. Black mushroom (photography by Elsa Bayart). Note the presence of a secondary buckling instability at the edge of the mushroom. This instability is also observed at the margin of flowers and other plants (see Audoly \& Boudaoud (2003) for example).

growth distribution. Indeed, in the footsteps of D'Arcy Thompson, our purpose is "to correlate with mathematical statement and physical law certain of the simpler outward phenomena of organic growth and structure or form". Relevency of the model proceeds from the fact that only simple growth laws are needed to induce non-trivial shapes. As a consequence, only simple organisms, like plants, algae, mushrooms and maybe jellyfish can be compared to our predictions. In the two examples treated in this paper, only the anisotropic property of the growth process is necessary for instabilities to occur and there is no need for complex spatial distributions of the cumulative growth rates. This is consistent with the large fluctuations characteristic of biological systems. However, this theory has several drawbacks. In all generality, the time dependence of the growth process can be included. Indeed, the adiabatic approximation allows us to treat the elastic response as instantaneous since mechanical time scales are much smaller than the characteristic time scale associated to growth. However the relation between the cumulative growth tensor (that is over a finite period of time) and the incremental growth tensor (describing an infinitesimal growth process) is still unclear. This is associated with the process of iteration of the multiplicative decomposition which is a complex mathematical operation. Cumulative growth requires to compute the stress tensor at each step and (virtually) destressing the sample before applying another growth increment (which is only defined on a stress-free state). Due to the complexity of three-dimensional elasticity however, resolution using this procedure is unpracticable. Fortunately, when the growth tensor is diagonal, the elastic and growth processes commute and the cumulative growth and elastic tensor are the product of their incremental couterparts.

\section{References}

Ashe, H. L. and Briscoe, J., 2006. The interpretation of morphogen gradients. Development, 133(3), 385-394.

Audoly, B., 1999. Stability of straight delamination blisters. Phys. Rev. Lett. 83, 41244127. 
Audoly, B., Boudaoud, A., 2003. Self-Similar Structures near Boundaries in Strained Systems. Phys. Rev. Lett. 91, 086105.

Ambrosi, D., Mollica, F., 2002. On the mechanics of a growing tumor. Int. J. Eng. Science 40(12),1297-1316.

Ben Amar, M., Pomeau, Y., 1997. Crumpled paper. Proc. Roy. Soc. A 453,729-755.

Ben Amar, M., Goriely, A., 2005. Growth and instability in elastic tissues. J. Mech. Phys. Solids 53, 2284-2319.

Cerda, E., Mahadevan, L., 1998. Conical Surfaces and Crescent Singularities in Crumpled Sheets. Phys. Rev. Lett. 80, 2358.

Ciarlet, P.G., 1980. A justification of the von Kármán equations. Arch. Rat. Mech. Analysis 73, 349.

Ciarletta, P., Micera, S., Accoto, D., Dario, P., 2006. A novel microstructural approach in tendon viscoelastic modeling at the fibrillar level. J. Biomech 39 2034-2042.

Ciarletta, P., Dario, P., Micera, S., 2008. Pseudo-hyperelastic model of tendon hysteresis from adaptative recruitment of collagen type I fibrils. Biomat 29 764-770.

Ciarletta, P., Ben Amar, M., 2009. A finite dissipative theory of temporary interfibrillar bridges in the extracellular matrix of ligaments and tendons. J. R. Soc. Interface.

Coen, E., Rolland-Lagan, A.G., Matthews, M., Bangham, J. A., and Prusinkiewicz, P., 2004. The Genetics of Geometry. Proc. Natl. Acad. Sci. 101, 4728-4735.

Cowin, S.C., Hegedus, D.H., 1976. Bone remodeling I: A theory of adaptive elasticity. J. Elasticity 6, 313-325.

Cowin, S.C., 1983. The mechanical and stress adaptive properties of bone. Ann. biomech. Engng. 11, 263-295.

Cruywagen, G.C., Maini, P.K., Murray, J.D., 1992. Sequential pattern formation in a model for skin morphogenesis, IMA J. Math. Appl. Med. Biol., 9, 227-248.

Cruywagen, G.C., Maini, P.K., Murray, J.D. 1997. Biological pattern formation on twodimensional domains: A nonlinear bifurcation analysis. SIAM Journal of Applied Mathematics, 57 (6), 1485-1509.

Dervaux, J., Ben Amar, M., 2008. Morphogenesis of growing soft tissues. Phys. Rev. lett. $101,068101$.

Dervaux, J., Ciarletta, P., Ben Amar, M., 2009. Morphogenesis of thin hyperelastic plates: A constitutive theory of biological growth in the Föppl-von Kármán limit. J. Mech. Phys. Solids. 57, 458-471.

Drozdov, A.D., Khanina, H., 1997. A model for the volumetric growth of soft tissue. Math. and comp. modelling 25, 11-29.

Dumais, J., 2007. Can mechanics control pattern formation in plants? Curr. Opin. plant. biol. 10, 58-62.

Efrati, E., Sharon, E., Kupferman, R., 2008. Elastic theory of unconstrained non-euclidian plates. preprint.

Entov, V.M., 1983. Mechanical model of scoliosis. IMech. Solids 18, 199-206.

Erbay, H.A., 1997. On the asymptotic membrane theory of thin hyperelastic plates. Int. J. Engng Sci. 35, 151-170.

Föppl, A., 1907. Vorlesungen über technische Mechanik, Leipzig.

Fung, Y.C., 1990. Biomechanics: motion, flow, stress, and growth. Springer, New York.

Fung, Y.C., 1993. Biomechanics: material properties of living tissues. Springer, New York. 
Goriely, A., Ben Amar, M., 2005. Differential growth and instability in elastic shells. Phys. Rev. Lett. 94, 198103-198107.

Goriely, A., Destrade, M., Ben Amar, M., 2006. Instabilities in elastomers and in soft tissues. Quart. Journ. mech. and appl. math. 59, 615-630.

Goriely, A., Ben Amar, M., 2007. On the definition and modeling of incremental, cumulative and continuous growth laws in morphoelasticity. Biomech. Model in mechanobiology 6, 289-296.

Gould, S.J., 1971. D’Arcy Thompson and the Science of Form. New Literary History, 2(2), 229-258.

Green, P.B., 1996. Transductions to Generate Plant Form and Pattern: An Essay on Cause and Effect. Annals of Botany 78, 269-28.

Guven, J., Müller, M.M., 2008. How paper folds: bending with local constraints. J. Phys. A: Math. Theor. 41(5), 055203.

Haughton, D.M., 2001. Elastic membranes In Finite Elasticity: Theory and Applications (ed. (R.W. Ogden, Y-B Fu) Ch. 7, pp. 233-Cambridge University Press.

Hoger, A., 1999. An incremental theory of volumetric growth for soft biological tissues. 1999 ASME Mechanics and Materials Conference 11, University of Virginia, Blacksburg, Virginia, June 27-30.

Holzapfel, G.A., Gasser, C.T., Ogden, R.W., 2000. A new Constitutive framework for arterial wall mechanics and a comparative study of material models J. Elasticity 61, $1-48$.

Hsu, F.H., 1968. The influences of mechanical loads on the form of a growing elastic body. J. Biomech. 1, 303-311.

Humphrey, J.D., Rajagopal, K.R., 2002. A constrained mixture model for growth and remodeling of soft tissues. Math. Mod. Meth. Appl. Sci. 12, 407430.

Humphrey, J.D., 2003. Continuum biomechanics of soft biological tissues. Proc. Roy. Soc. Lond. A 459, 3-46.

Klein, Y., Efrati, E., Sharon, E., 2007. Shaping of elastic sheets by prescription of NonEuclidian metrics. Science 315, 1116-1120.

Kondo, S., 2002. The reaction-diffusion system: a mechanism for autonomous pattern formation in the animal skin, Genes to Cells 7, 535-541.

Landau, L., Lifchitz, E., 1990. Théorie de l'elasticité, Mir, Moscow.

Lubarda, V.A., 2004. Constitutive Theories Based on the Multiplicative Decomposition of Deformation Gradient: Thermoelasticity, Elastoplasticity and Biomechanics. Appl. Mech. Rev. 57, 95-108.

Marder, M., Papanicolaou, N., 2006. Geometry and elasticity of strips and Flowers. Journ Stat. Phys. 125, 5-6, 1069-1096.

Marder, M., Deegan, R.D., Sharon, E., 2007. Crumpling,buckling and cracking: Elasticity of thin sheets. Phys. Today 60 33-38.

Müller, M.M., Ben Amar, M., Guven, J., 2008. Conical defects in growing sheets. Phys. Rev. Lett. 101, 156104

Naghdi, P.M., 1990 A critical review of the state of finite plasticity. Zeitschrift für angewandte Mathematik und Physik 41(3), 315-394.

Newell, A.C., Shipman, P.D., 2005. Plants and Fibonacci. J. Stat. Phys. 121, 937-968.

Nichols, W.W., O'Rourke, M.F., 1998. McDonald's Blood Flow in Arteries, 4th edn. London: Arnold. 
Nüsslein-Volhard, C., Wieschaus, E., 1980. Mutations affecting segment number and polarity in Drosophila. Nature 287, 795-801.

Ogden, R.W., 1997. Non-linear elastic deformations, Dover, New York.

Rodriguez, A.K., Hoger, A., and McCulloch, A., 1994. Stress-dependent finite growth in soft elastic tissue. J. Biomech. 27, 455-467.

Rogulja, D., Irvine, K.D. 2005 Regulation of cell proliferation by a morphogen gradient. Cell, 123, 449-461.

Rolland-Lagan, A.G., Bangham, J.A., Coen, E., 2003. Growth dynamics underlying petal shape and asymmetry Nature 422, 161-163.

Roy, C., 1880. The elastic properties of the arterial wall. Phil. Trans. R. Soc. Lond. B 99, 1-31.

Serikawa, K.A., Mandoli, D.F., 1998 An analysis of morphogenesis of the reproductive whorl of Acetabularia acetabulum. Planta 207, 96104.

Sharon, E., Roman, B., Marder, M., Shin, S.H., Swinney, H.L., 2002. Mechanics: Buckling cascades in free sheets. Nature 419, 519-580.

Skalak, R., Tozeren, R.P. Zarda, and Chien, S., 1973. Strain energy function of red blood cell membranes. Biophys. J. 13, 245-264.

Skalak, R., 1981. Growth as a finite displacement field. in Carlson, D. E. and Shield, R. T. (eds), Proceedings of the IUTAM Symposium on Finite Elasticity., Martinus Nijhoff, The Hague.

Skalak, R., Hoger, A., 1997. Kinematics of surface growth. J. Math. Biol. 35, 869-907.

Stein, A.A., 1995. The deformation of a rod of growing biological material under longitudinal compression. J. Appl. maths. Mech. 59, 139-146.

Struhl, G., Struhl, K., Macdonald, P. M., 1989. The gradient morphogen bicoid is a concentration-dependent transcriptional activator. Cell 57, 1259-1273.

Taber, L., 1995. Biomechanics of growth, remodeling and morphogenesis. Appl. Mech. Rev. 48, 487-545.

Thompson, D.W., 1992. On growth and form: The complete revised edition Dover, New York.

Treloar, L.R.G., 1975. The physics of rubber elasticity, 3rd edn. Oxford University.

von Kármán, T., 1907. Enzyclopädie der mathematischen Wissenschaften, Forschungsarabeiten Berlin.

Wertheim, M.G., 1847. Mémoire sur l'eastocote et la cohésion des principaux tissues du corps humain. Ann. Chim. Phys. 21, 385-414.

White, R.J., Nie, Q., Lander, A.D., Schilling, T.F., 2007. Complex Regulation of cyp26a1 Creates a Robust Retinoic Acid Gradient in the Zebrafish Embryo. PLoS Biol 5(11): e304.

Wolpert, L., 1996. One hundred years of positional information. Trends Genet. 12, 359-364. 